\title{
Comparison of digital and silicone impressions for single-tooth implants and two- and three-unit implants for a free-end edentulous saddle
}

Koudai Nagata ${ }^{1}$, Kei Fuchigami ${ }^{1}$, Yurie Okuhama ${ }^{1}$, Kana Wakamori ${ }^{1}$, Hayato Tsuruoka ${ }^{1}$, Toshifumi Nakashizu ${ }^{2}$, Noriyuki Hoshi ${ }^{3}$, Mihoko Atsumi ${ }^{3}$, Katsuhiko Kimoto ${ }^{3}$ and Hiromasa Kawana ${ }^{\text {* }}$

\begin{abstract}
Background: The use of intraoral scanners (IOS) has facilitated the use of digital workflows for the fabrication of implant-supported prostheses not only for single missing teeth, but also for multiple missing teeth. However, the clinical application of IOS and computer-aided design/manufacturing (CAD/CAM) in implant-supported prosthodontics remains unclear. This study aimed to compare the accuracy of digital and silicone impressions for single-tooth implants for bounded edentulous spaces and two-unit and three-unit implant-supported fixed dental prostheses for free-end edentulous spaces.
\end{abstract}

Methods: This study enrolled 30 patients ( $n=10$ for each of the three groups) with an average age of 61.9 years. Conventional silicone-based and digital IOS-based impressions were made for all patients, and the implant superstructures were fabricated. We measured the scan-body misfit and compared the accuracy of the impressions for single-unit, two-unit, and three-unit implant prostheses with a bounded edentulous space by superimposing the standard triangulated language (STL) data obtained from IOS over the STL data of the plaster model used for final prosthesis fabrication. The scan bodies of the superimposed single-molar implant, two-unit implant prosthesis without teeth on the mesial side, two-unit implant prosthesis without teeth on the distal side, three-unit implant prosthesis without teeth on the mesial side, and three-unit implant prosthesis without teeth on the distal side were designated as A, B1, B2, C1, and C2, respectively. The misfit for each scan body was calculated and the accuracies were compared using the Tukey-Kramer method.

Results: The average scan-body misfit for conditions A, B1, B2, C1, and C2 was $40.5 \pm 18.9,45.4 \pm 13.4,56.5 \pm 9.6$, $50.7 \pm 14.9$, and $80.3 \pm 12.4 \mu \mathrm{m}$, respectively. Significant differences were observed between the accuracies of $A$ and $B 2, A$ and $C 2$, and $C 1$ and $C 2(P<0.001)$.

Conclusions: IOS and CAD/CAM can find clinical applications for implant-supported prostheses of up to three units for a bounded edentulous saddle. The use of IOS could render implant treatment easier, benefiting both the surgeons and patients. Prosthesis maladjustment may lead to peri-implantitis and prosthetic fracture. Therefore, further

\footnotetext{
*Correspondence: kawana@kdu.ac.jp

1 Department of Oral and Maxillofacial Implantology, Kanagawa Dental

University, 82 Inaoka-cho, Yokosuka 238-8580, Japan

Full list of author information is available at the end of the article
} original author(s) and the source, provide a link to the Creative Commons licence, and indicate if changes were made. The images or other third party material in this article are included in the article's Creative Commons licence, unless indicated otherwise in a credit line to the material. If material is not included in the article's Creative Commons licence and your intended use is not permitted by statutory regulation or exceeds the permitted use, you will need to obtain permission directly from the copyright holder. To view a copy of this licence, visit http://creativecommons.org/licenses/by/4.0/. The Creative Commons Public Domain Dedication waiver (http://creativecommons.org/publicdomain/zero/1.0/) applies to the data made available in this article, unless otherwise stated in a credit line to the data. 
validation of the accuracy of IOS impressions is required in patients with multiple missing teeth in long-span implant prostheses.

Keywords: Dental implant, Digital impression, Digital workflow, Implant impression, Intraoral scanner

\section{Background}

The use of digital technology in clinical dentistry has become widespread in recent years, enabling dentists to provide better care to their patients. The use of intraoral scanners (IOS) and computer-aided design/manufacturing $(\mathrm{CAD} / \mathrm{CAM})$ systems for the fabrication of crowns and bridges has facilitated the digitization of almost all the processes involved in prosthodontic treatment [1-3].

The use of IOS has facilitated the use of digital workflows for the fabrication of implant-supported prostheses not only for single missing teeth, but also for multiple missing teeth $[4,5]$. The benefits of using IOS include reduced patient discomfort, shorter chair time, shorter prosthesis fabrication time, and the ability to store patients' biometric information as data [6, 7]. However, only a few clinical studies have reported on the utility of IOS for implant-supported prosthetic treatment. Most previous studies conducted in vitro comparisons of precision and accuracy between the digitally fabricated and conventional models used for implant-supported prosthetic treatment [8-10]. Kocaağaoğlu et al. [11] compared the marginal misfit of three-unit frames made with conventional and digital impressions and found that the mean marginal misfit of conventional impressions was $98.8 \pm 16.43 \mu \mathrm{m}$, whereas that of the digital impression was $65.14 \pm 18.05 \mu \mathrm{m}$. The authors reported that the misfit was larger with the conventional method. The extent to which IOS and CAD/CAM can be used clinically for the fabrication of implant-supported prostheses remains unclear.

In clinical practice, it is impossible to ascertain the impression method with the highest accuracy when comparing the traditional silicone method with the digital method. Thus, evaluating the misfits of the prostheses fabricated with each method affords the only opportunity to verify their respective accuracies. The purpose of this study was to compare the clinical accuracy of digital and silicone impressions made for single-tooth implants with teeth present on both sides and two- and three-unit implant-supported fixed dental prostheses with teeth present only on one side of the edentulous space.

\section{Methods}

\section{Study design}

This study was approved by the ethics committee of Kanagawa Dental University (approval number 555). Research involving human participants, were performed in accordance with the Declaration of Helsinki and with the relevant guidelines and regulations. Written informed consent was obtained from all participants. Digital IOS-based impressions and conventional silicone-based impressions were made for all patients, and the implant superstructures were fabricated (Fig. 1). The accuracy of the impressions made for single-tooth implants with teeth present on both sides and two-unit or three-unit implant-supported fixed dental prostheses with teeth present only on one side of the edentulous space was compared by superimposing the standard triangulated language (STL) data obtained from the IOS and the STL data from plaster models retrieved from the silicone impressions.

\section{Participants}

Thirty patients, with an average age of 61.9 years, who agreed to undergo implant treatment at our university hospital, were included according to the following three criteria: missing single molar with natural teeth on both sides $(n=10)$, two missing teeth with natural teeth present on only one side of the edentulous space $(\mathrm{n}=10)$, and three missing teeth with natural teeth present on only one side of the edentulous space $(n=10)$. All patients were treated by the same dentist from the Department of Oral and Maxillofacial Implantology, and all laboratory procedures were performed by the same dental technician.

Patients were included in the study only if they did not have a history of systemic diseases, were non-smokers, and did not require bone grafting.

\section{Surgical procedure}

All implant placements were performed via freehand insertion and in accordance with the implant system protocol [12]. The Straumann ${ }^{\circledR} \phi 4.1 \mathrm{~mm}$ (standard plus implant, bone level tapered implant, Basel, Switzerland) implant system was used in this study. All implant surgeries were performed using the two-stage method, and the post-surgical recovery period was 2 months.

\section{Evaluation and measurement}

The open tray (made of resin) technique with siliconebased impression material was used as a conventional method to acquire STL data. An impression coping was placed over the implant in the oral cavity, and precise impressions were made using a silicone-based material 


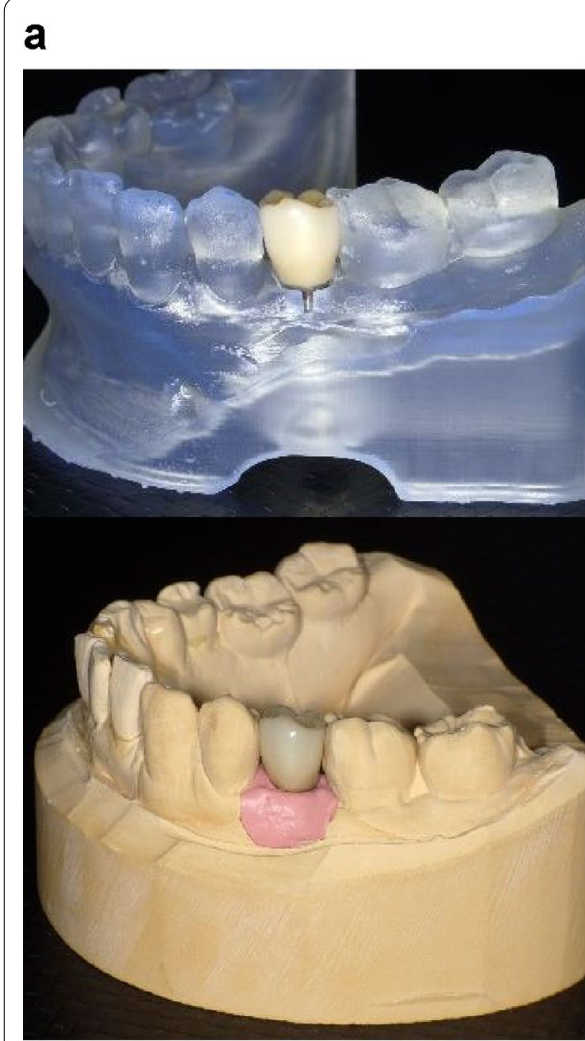

b

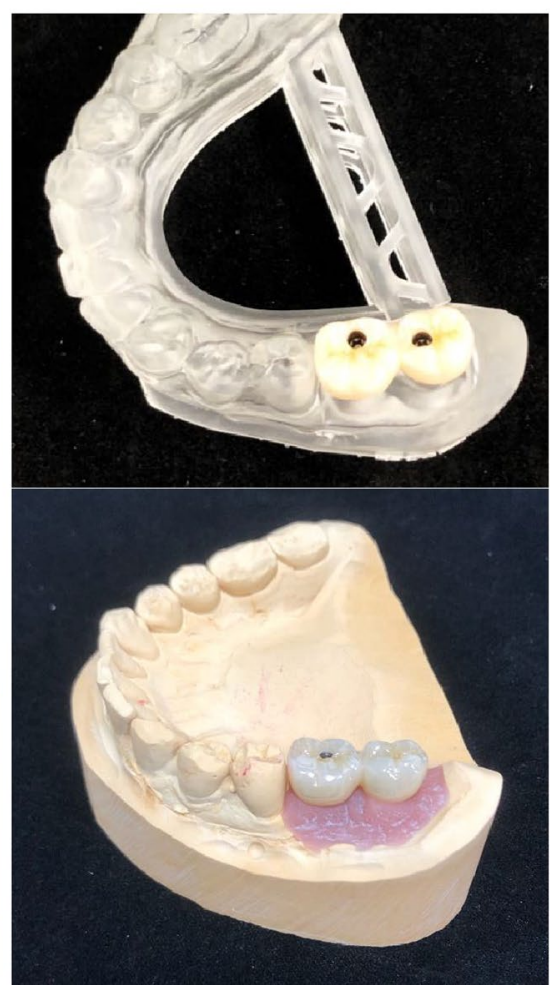

C

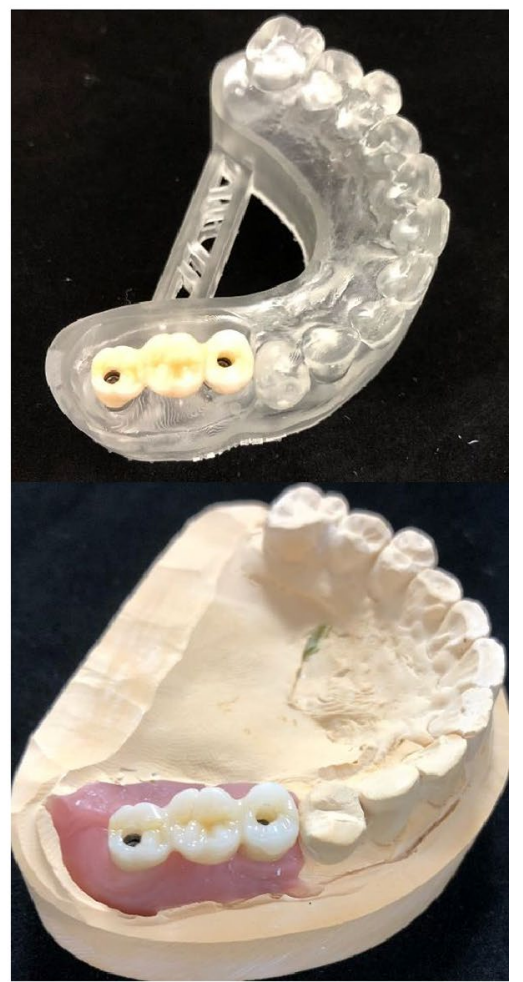

Fig. 1 The superstructures were fabricated from impressions obtained using an intraoral scanner (IOS) and silicone material. a Single-tooth implants. b Two-unit implants. c Three-unit implants

(Aquasil Ultra $^{\circledR}$; Dentsply Sirona, York, PA). Subsequently, a scan body (Mono Scanbody RC, RN, Straumann ${ }^{\circledR}$, Basel, Switzerland) was mounted over the plaster model (New Fujirock ${ }^{\circledR}$; GC, Tokyo, Japan), followed by scanning with a 3D scanner (Ceramill Map400 ${ }^{\circledR}$; Amann Girrbach, Vienna, Austria), and conversion to STL data. For STL data acquisition by digital impression technique, a scan body was placed in the patient's mouth and digital impressions were taken using an IOS (Trios $3^{\circledR}$, 3Shape; Copenhagen, Denmark). Using Geomagic Control ${ }^{\circledR}$ (3D Systems, Washington, DC), the STL data obtained from the conventional method and the STL data obtained from the digital impression method were superimposed to measure the scan body misfit. The superposition of the two STL data sets was performed after trimming the excess data, followed by manual alignment based on three landmarks, and best-fit registration was used for greater accuracy. Three points were randomly selected from the data of the superimposed scan body, and the average value was considered as the misfit between the digital and conventional impressions. The positions of the scan body mounted on the plaster model and scan body mounted in the oral cavity were set to be equivalent.
CAD software (Exocad ${ }^{\circledR}$; Exocad, Berlin, Germany) and CAM (Ceramill motion $2^{\circledR}$; Amann Girrbach, Wien, Austria) were used to design and fabricate the superstructure, respectively (Fig. 2).

A fixed screw-retained implant superstructure composed of zirconia (Ceramill Zolid ${ }^{\circledR}$, Amann Girrbach, Vienna, Austria) was used. All silicone and IOS impressions were made at the implant level. The abutment was not interposed with the superstructure in single implants, while the abutments were interposed with the superstructure in implants with two or three units. All superstructures were fabricated with zirconia.

The superimposed images of the scan bodies in patients with single-tooth molar implants bound by teeth on the mesial and distal sides, were designated as A. The superimposed images of the scan bodies in patients with two-unit implant prosthesis with no teeth on the mesial side were designated as B1, and those without teeth on the distal side were designated as B2. The superimposed images of the scan bodies in patients with three-unit implant prosthesis without teeth on the mesial side were designated as $\mathrm{C} 1$ and those without teeth on the distal side were designated as $\mathrm{C} 2$. The misfit for each scan body 


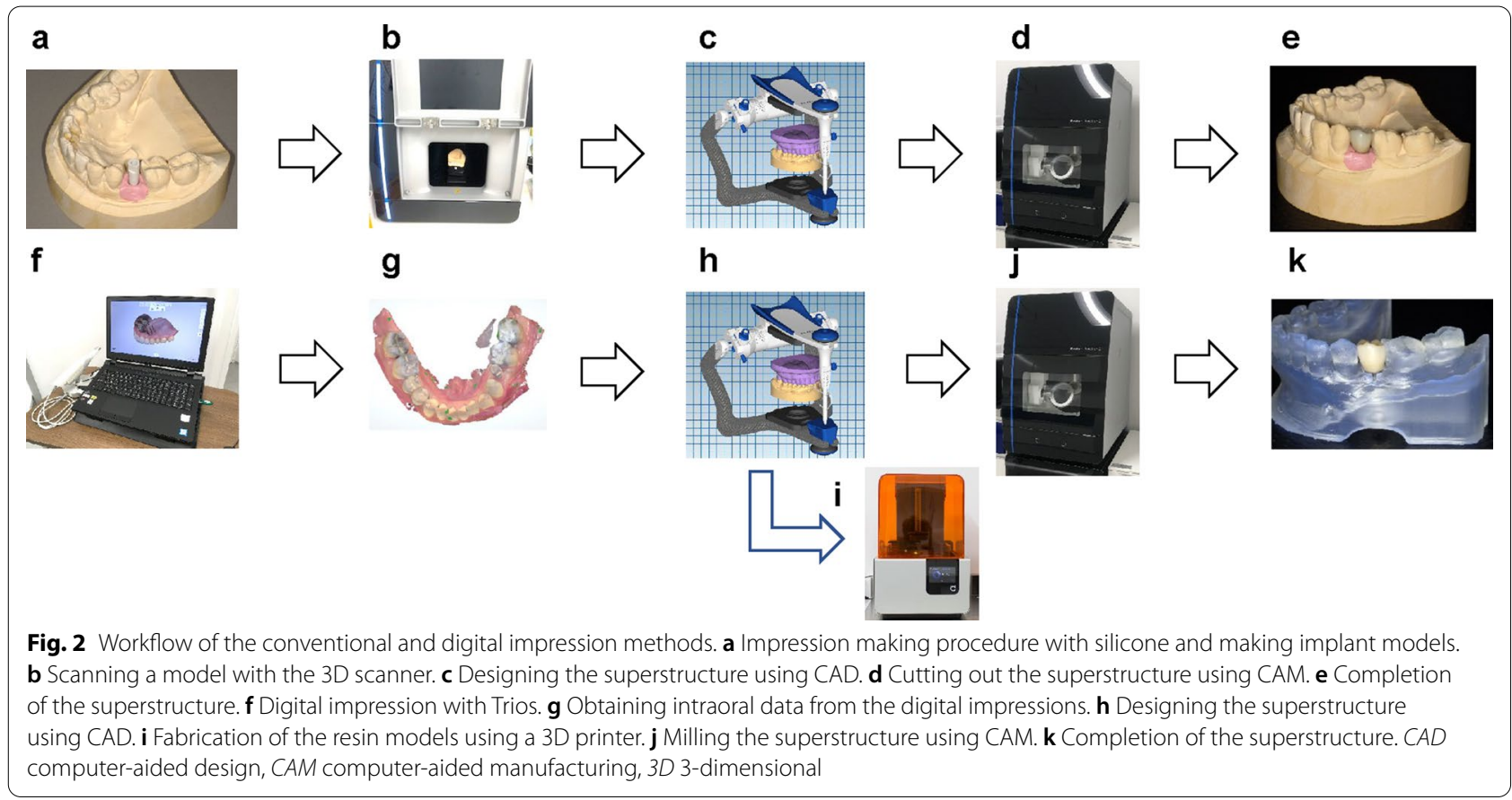

was calculated (Fig. 3) and the accuracies were subsequently compared.

The implantation sites of two-unit prostheses included the second premolar and first molar, or the first molar and second molar, while the sites of three-unit prostheses were the first premolar, first molar/second premolar, and the second molar. The average distance between the centers of the implants was $9.9 \mathrm{~mm}$ in the two-unit prostheses and $15.3 \mathrm{~mm}$ in the three-unit prostheses.

\section{Statistical analysis}

G-Power (version 3.1.9.2) was used to perform the oneway analysis of variance. We calculated the sample size required to obtain $80 \%$ of the effect size of 0.4 at $\alpha=0.05$. A total sample size of 60 people was needed. However, since there were not many patients who met the requirements, the total sample size was set at 30 in this study.

We considered a P-value of $<0.05$ to indicate significance. BellCurve for Excel (Social Survey Research Information Co., Ltd., Tokyo, Japan) was used to perform statistical processing using the Tukey-Kramer method.

\section{Results}

\section{Misfits in scan bodies}

The average misfit was calculated in all the conditions. The average misfit in conditions A, B1, B2, C1, and C2 was $40.5 \pm 18.9,45.4 \pm 13.4,56.5 \pm 9.6,50.7 \pm 14.9$, and $80.3 \pm 12.4 \mu \mathrm{m}$, respectively.

\section{Comparison of scan-body misfits between the different conditions}

The misfits in the different conditions were analyzed as a measure of accuracy for the comparison among the different conditions. A significant difference was observed between $\mathrm{A}$ and $\mathrm{B} 2$, and between $\mathrm{A}$ and $\mathrm{C} 2(\mathrm{P}<0.001)$. The degree of misfit was the highest in $\mathrm{C} 2$ when compared to all the other conditions (Fig. 4).

\section{Discussion}

This study compared the accuracy of digital impressions made using IOS with that of conventional impressions made using silicone impression materials. The results showed that the scan-body misfit between the two methods was the highest for $\mathrm{C} 2$, while condition $\mathrm{A}$ had the smallest misfit.

The current implant-supported prosthodontic treatment protocol using silicone impressions entails the placement of the impression coping in the oral cavity, followed by the acquisition of the silicone-based impression. Subsequently, a plaster model is retrieved from the impression and the scan body is placed on the plaster model, which is then read by a 3D scanner, followed by the CAD/CAM process. Although the fabrication of zirconia superstructures is predominantly performed using this method, there have been concerns that the deformation of the silicone impression material and expansion of the plaster model could affect the accuracy of the prosthesis [13-15]. 


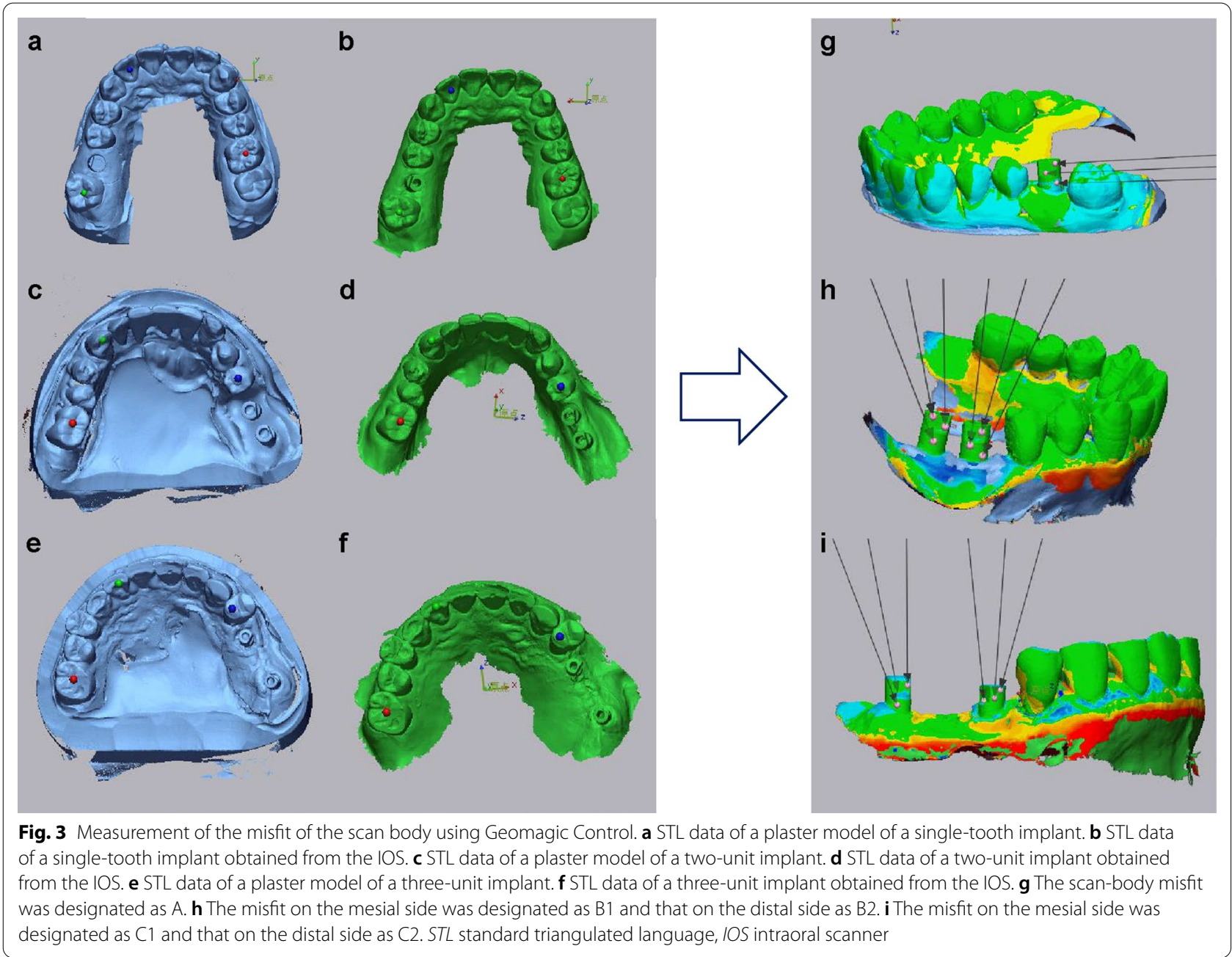

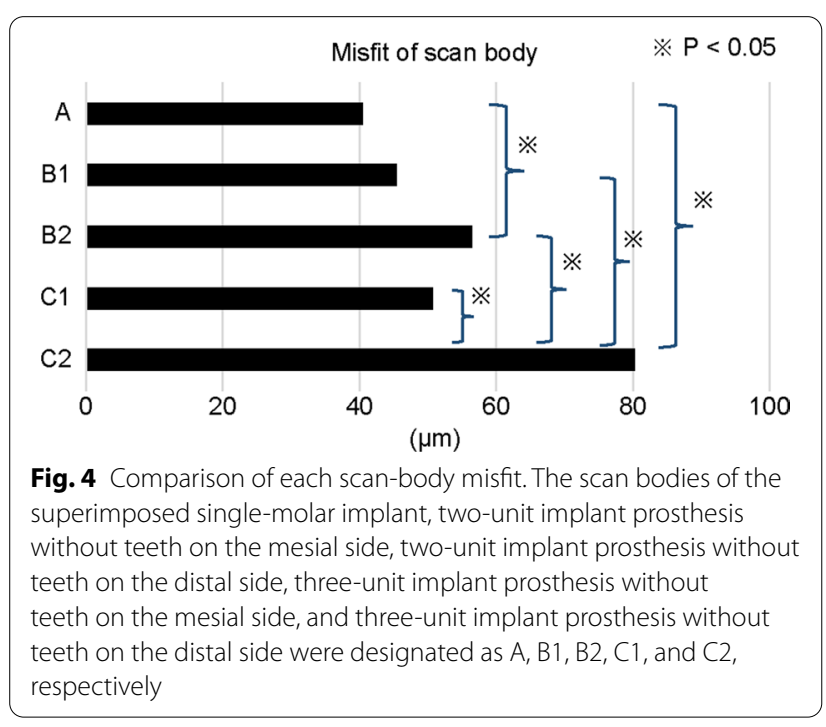

However, digital impressions using IOS have facilitated the designing of zirconia superstructures using $C A D$ and machining using CAM [16, 17], which have streamlined the process of prosthesis fabrication; thus, reducing the treatment time, laboratory time, and errors $[8,18]$. Several studies have reported on the performance of IOSs. Seelbach et al. [1] compared the digital and conventional impression techniques for crowns and bridges and found no difference among the fit of crown margins fabricated using the three types of IOSs (Lava Chairside Oral Scanner, Chairside Economical Restoration of Esthetic Ceramic, and iTero) and the conventional method. Systematic reviews conducted by Chochlidakis et al. [19] and Ahlholm et al. [20] have also found that the fit of prostheses such as crowns and bridges fabricated from conventional or digital impressions was good, and no difference was observed between them. Flügge et al. [21] conducted a systematic review of 79 studies on implant impression making and found that only 20 studies focused on digital impressions, while most studies used plaster or other 
models. Fukazawa et al. [22] placed a ball attachment on study models and measured the respective accuracies of the IOS and laboratory scanner in a study that investigated the accuracy of IOSs in implant treatment. The misfit of the IOS was greater in the model with a distance of $18.4 \mathrm{~mm}$ between the centers of the ball attachments than that in the model with a distance of $9.6 \mathrm{~mm}$ between the centers of ball attachments. Flügge et al. [23] also evaluated the IOS using study models and reported that the accuracy of the IOS decreased with the increase in the distance and angle between the scan bodies. Tan et al. [24] also reported that a short interdental distance between implants was related to higher accuracy using a maxillary edentulous model. All of the above-mentioned studies were based on models and reported a decrease in accuracy as the distance between the implant bodies increased.

Our results showed that there were no significant differences between $\mathrm{A}$ and $\mathrm{B} 1$ or $\mathrm{A}$ and $\mathrm{C} 1$, whereas significant differences were observed between $\mathrm{A}$ and $\mathrm{B} 2$ and between $\mathrm{A}$ and $\mathrm{C} 2$. Condition $\mathrm{A}$, where the mesial and distal teeth adjacent to the (edentulous) defect were present, showed the smallest misfit, followed by B1 and $\mathrm{C} 1$, which were located close to the remaining teeth. Conversely, the misfit was larger in $\mathrm{B} 2$ and $\mathrm{C} 2$, where the distance from the landmark was greater. The results were similar to those reported by previous studies. Few clinical studies have reported on the accuracy of digital impressions in implant treatment. Delize et al. [25] compared superstructures fabricated using digital impressions and the conventional method based on the occlusal and interdental contact in 31 patients with a single missing tooth. They reported that the respective fits of the superstructures fabricated by both processes were good and lacked a significant difference. Mühlemann et al. [26] measured the accuracy of impressions in five patients with a single missing tooth with teeth on both sides (of the edentulous space) using the conventional method and three types of IOSs (iTero Cadent, Lava True Definition, and Trios 3Shape). They reported that the scan-body misfit was $32.7 \pm 11.6 \mu \mathrm{m}$ using the conventional method, and $57.2 \pm 32.6$ and $88.6 \pm 46 \mu \mathrm{m}$ using the iTero and Trios systems, respectively. Gedrimiene et al. [27] compared conventional silicone-based and IOS-based digital impressions in six patients with multiple missing teeth with teeth on both sides of the edentulous space and found that the misfit of the scan body was $70.8 \pm 59 \mu \mathrm{m}$ and that IOSs can be used in implant-supported prostheses with up to four units. Studies investigating the prosthetic fit have reported that cement spaces lower than $100 \mu \mathrm{m}$ were acceptable [28-30]. Al-Meraikhi et al. [31] investigated the fit of the abutment on the implant body and reported that spaces lower than $135 \mu \mathrm{m}$ were acceptable.
In the present study, the average misfit for three-unit prostheses with no remaining teeth on the distal side was found to be $80.3 \pm 12.4 \mu \mathrm{m}$. We suggest that digital impressions using IOS could be indicated in clinical practice for implant prostheses of up to three units, based on the findings of the current and previous studies. The greater the angular error of the implant body, the lower the accuracy of the IOS impression; therefore, guided implant surgery is among the methods that can ensure accurate prosthetic treatment. The fit of the superstructure in implant prostheses is likely to vary in clinical practice depending on various factors, such as the condition of the edentulous space, the number of units in the prosthesis, and whether powder was used when taking impressions with IOS.

Maladjustment of the prosthesis may lead to periimplantitis and prosthetic fracture. Therefore, further validation of the accuracy of IOS impressions is required in patients with multiple missing teeth.

A limitation of this study is the small sample size for each condition; the eligibility criteria made it difficult to include more patients in each group. Another limitation is that the bone resorption, loosening of the superstructure, and failure of the superstructure for each impression type could not be evaluated using follow-up examinations.

Finally, a single implantologist was in charge of the entire process from implant placement to prosthetic treatment. After placement of the superstructure, maintenance was performed every three or six months. For patient self-care, he recommended thorough brushing and chlorhexidine mouthwash. Owing to the current world situation, the medical community is baffled by the coronavirus disease 2019 (COVID-19) pandemic. Severe acute respiratory syndrome coronavirus 2 adheres to the oral cavity and respiratory tract, and dental treatment may increase the risk of infection; therefore, knowledge regarding its prevention is necessary [32,33]. It is well known that chlorhexidine is effective in the treatment of gingivitis and the prevention of peri-implantitis [34]. There are also reports that chlorhexidine exerts a preventive effect on COVID-19 [35]. It may be possible to reduce the risk of infection for both the dentist and patient by having patients wash their mouths with chlorhexidine before treatment. In addition, thorough infection prevention in medical care systems can be a measure against not only COVID-19 but also various other infectious diseases [36]. Whether digital technologies, such as IOS, can reduce the risk of infection compared to conventional methods will be a subject of future research.

\section{Conclusions}

The application of IOS for implant treatment could render treatment easier, which is beneficial for both surgeons and patients. However, its application in 
implant-supported prostheses has been investigated by only a few clinical studies; thus, the extent to which digital technologies, such as IOS, can be applied in routine clinical practice is unclear. Our study suggested that digital impressions made using IOS can be used for the fabrication of implant prostheses of up to three units with teeth present on only one side of the edentulous space. We will endeavor to verify the clinical results in long-span implant prostheses in the future.

\section{Abbreviations}

3D: Three-dimensional; CAD/CAM: Computer-aided design/manufacturing; IOS: Intraoral scanners; STL: Standard triangulated language.

\section{Acknowledgements}

Not applicable.

\section{Authors' contributions}

HK, MA conceived the idea and designed the study. KN performed the research. $\mathrm{KW}, \mathrm{YO}, \mathrm{TN}$, and $\mathrm{HT}$ analyzed the data. $\mathrm{KK}, \mathrm{NH}$, and $\mathrm{KF}$ contributed with new methods or models. KN wrote the paper. All authors read and approved the final manuscript.

\section{Funding}

This study did not receive funding from external sources.

\section{Availability of data and materials}

The datasets obtained and analyzed during the current study are available from the corresponding author upon reasonable request.

\section{Declarations}

\section{Ethics approval and consent to participate}

This study was approved by the Kanagawa Dental University Ethics Committee (approval number 555). Written informed consent was obtained from all patients.

\section{Consent for publication}

Not applicable.

\section{Competing interests}

The authors declare that they have no competing interests.

\begin{abstract}
Author details
${ }^{1}$ Department of Oral and Maxillofacial Implantology, Kanagawa Dental University, 82 Inaoka-cho, Yokosuka 238-8580, Japan. ${ }^{2}$ Department of Dental Laboratory, Kanagawa Dental University Hospital, Yokosuka, Japan. ${ }^{3}$ Division of Prosthodontics and Oral Implantology, Department of Oral Interdisciplinary Medicine, Graduate School of Dentistry, Kanagawa Dental University, Yokosuka, Japan.
\end{abstract}

Received: 1 July 2021 Accepted: 16 September 2021 Published online: 23 September 2021

\section{References}

1. Seelbach P, Brueckel C, Wöstmann B. Accuracy of digital and conventional impression techniques and workflow. Clin Oral Investig. 2013;17:1759-64.

2. Berrendero S, Salido MP, Ferreiroa A, Valverde A, Pradíes G. Comparative study of all-ceramic crowns obtained from conventional and digital impressions: clinical findings. Clin Oral Investig. 2019;23:1745-51.
3. Boeddinghaus M, Breloer ES, Rehmann P, Wöstmann B. Accuracy of single-tooth restorations based on intraoral digital and conventional impressions in patients. Clin Oral Investig. 2015;19:2027-34.

4. Lo Russo L, Caradonna G, Biancardino M, De Lillo A, Troiano G, Guida L. Digital versus conventional workflow for the fabrication of multiunit fixed prostheses: a systematic review and meta-analysis of vertical marginal fit in controlled in vitro studies. J Prosthet Dent. 2019;122:435-40.

5. Aktas G, Özcan N, Aydin DH, Şahin E, Akça K. Effect of digitizing techniques on the fit of implant-retained crowns with different antirotational abutment features. J Prosthet Dent. 2014;111:367-72.

6. Vandeweghe S, Vervack V, Dierens M, De Bruyn H. Accuracy of digital impressions of multiple dental implants: an in vitro study. Clin Oral Implants Res. 2017;28:648-53.

7. Borgonovo AE, Rigaldo F, Battaglia D, Re D, Giannì AB. Digital device in postextraction implantology: a clinical case presentation. Case Rep Dent. 2014;2014:327368.

8. Joda T, Brägger U. Time-efficiency analysis of the treatment with monolithic implant crowns in a digital workflow: a randomized controlled trial. Clin Oral Implants Res. 2016;27:1401-6.

9. Papaspyridakos P, Gallucci GO, Chen CJ, Hanssen S, Naert I, Vandenberghe B. Digital versus conventional implant impressions for edentulous patients: accuracy outcomes. Clin Oral Implants Res. 2016;27:465-72.

10. Camardella LT, Vilella OV, van Hezel MM, Breuning KH. Accuracy of stereolithographically printed digital models compared to plaster models. J Orofac Orthop. 2017;78:394-402.

11. Kocaağaoğlu H, Albayrak H, Cinel Sahin S, Gürbulak AG. Evaluation of marginal adaptation in three-unit frameworks fabricated with conventional and powder-free digital impression techniques. J Adv Prosthodont. 2019;11:262-70.

12. Toyoshima T, Wagner W, Klein MO, Stender E, Wieland M, Al-Nawas B. Primary stability of a hybrid self-tapping implant compared to a cylindrical non-self-tapping implant with respect to drilling protocols in an ex vivo model. Clin Implant Dent Relat Res. 2011;13:71-8.

13. Camardella LT, de Vasconcellos VO, Breuning H. Accuracy of printed dental models made with 2 prototype technologies and different designs of model bases. Am J Orthod Dentofacial Orthop. 2017;151:1178-87.

14. Zhang F, Suh KJ, Lee KM. Validity of intraoral scans compared with plaster models: an in-vivo comparison of dental measurements and 3D surface analysis. PLOS ONE. 2016;11: e0157713. https://doi.org/10.1371/journal. pone.0157713.

15. Mizumoto RM, Yilma B. Intraoral scan bodies in implant dentistry: a systematic review. J Prosthet Dent. 2018;120:343-52.

16. Lopez-Suarez C, Gonzalo E, Pelaez J, Serrano B, Suarez MJ. Marginal vertical discrepancies of monolithic and veneered zirconia and metalceramic three-unit posterior fixed dental prostheses. Int J Prosthodont. 2016:29:256-8

17. Bayramoglu E, Özkan YK, Yildiz C. Comparison of marginal and internal fit of press-on-metal and conventional ceramic systems for three- and fourunit implant-supported partial fixed dental prostheses: an in vitro study. J Prosthet Dent. 2015;114:52-8.

18. Kim TG, Kim S, Choi H, Lee JH, Kim JH, Moon HS. Clinical acceptability of the internal gap of CAD/CAM PD-AG crowns using intraoral digital impressions. Biomed Res Int. 2016;2016:7065454. https://doi.org/10.1155/ 2016/7065454.

19. Chochlidakis KM, Papaspyridakos P, Geminiani A, Chen CJ, Feng IJ, Ercoli C. Digital versus conventional impressions for fixed prosthodontics: a systematic review and meta-analysis. J Prosthet Dent. 2016;116:184-90. e12.

20. Ahlholm P, Sipilä K, Vallittu P, Jakonen M, Kotiranta U. Digital versus conventional impressions in fixed prosthodontics: a review. J Prosthodont. 2018;27:35-41.

21. Flügge T, van der Meer WJ, Gonzalez BG, Vach K, Wismeijer D, Wang P. The accuracy of different dental impression techniques for implantsupported dental prostheses: a systematic review and meta-analysis. Clin Oral Implants Res. 2018;29:374-92.

22. Fukazawa S, Odaira C, Kondo H. Investigation of accuracy and reproducibility of abutment position by intraoral scanners. J Prosthodont Res. 2017;61:450-9.

23. Flügge TV, Att W, Metzger MC, Nelson K. Precision of dental implant digitization using intraoral scanners. Int J Prosthodont. 2016;29:277-83. 
24. Tan MY, Yee SHX, Wong KM, Tan YH, Tan KBC. Comparison of threedimensional accuracy of digital and conventional implant impressions: effect of interimplant distance in an edentulous arch. Int J Oral Maxillofac Implants. 2019;34:366-80.

25. Delize V, Bouhy A, Lambert F, Lamy M. Intrasubject comparison of digital vs. conventional workflow for screw-retained single-implant crowns: prosthodontic and patient-centered outcomes. Clin Oral Implants Res. 2019;30:892-902.

26. Mühlemann S, Greter EA, Park JM, Hämmerle CHF, Thoma DS. Precision of digital implant models compared to conventional implant models for posterior single implant crowns: a within-subject comparison. Clin Oral Implants Res. 2018;29:931-6.

27. Gedrimiene A, Adaskevicius R, Rutkunas V. Accuracy of digital and conventional dental implant impressions for fixed partial dentures: a comparative clinical study. J Adv Prosthodont. 2019;11:271-9.

28. Shim JS, Lee JS, Lee JY, Choi YJ, Shin SW, Ryu JJ. Effect of software version and parameter settings on the marginal and internal adaptation of crowns fabricated with the CAD/CAM system. J Appl Oral Sci. 2015;23:515-22.

29. Paul N, Raghavendra Swamy KN, Dhakshaini MR, Sowmya S, Ravi MB. Marginal and internal fit evaluation of conventional metal-ceramic versus zirconia CAD/CAM crowns. J Clin Exp Dent. 2020;12:e31-7.

30. Görüş Z, Üner DD. Evaluation of marginal fits of crown substructure designs in implant-supported abutments. Med Sci Monit. 2018;24:7778-84
31. Al-Meraikhi H, Yilmaz B, McGlumphy E, Brantley W, Johnston WM. In vitro fit of CAD-CAM complete arch screw-retained titanium and zirconia implant prostheses fabricated on 4 implants. J Prosthet Dent. 2018;119:409-16.

32. Gherlone E, Polizzi E, Tetè G, Capparè P. Dentistry and Covid-19 pandemic: operative indications post-lockdown. New Microbiol. 2021;44:1-11.

33. Gherlone EF, Polizzi E, Tetè G, De Lorenzo R, Magnaghi C, Rovere Querini $P$, Ciceri F. Frequent and persistent salivary gland ectasia and oral disease after COVID-19. J Dent Res. 2021;100:464-71.

34. Polizzi E, Tetè G, Bova F, Pantaleo G, Gastaldi G, Capparè P, et al. Antibacterial properties and side effects of chlorhexidine-based mouthwashes. A prospective, randomized clinical study. J Osseointegr. 2020;12:2-7.

35. Vergara-Buenaventura A, Castro-Ruiz C. Use of mouthwashes against COVID-19 in dentistry. Br J Oral Maxillofac Surg. 2020;58:924-7.

36. Tecco S, Parisi MR, Gastaldi G, Polizzi E, D’Amicantonio T, Zilocchi I, et al. Point-of-care testing for hepatitis $C$ virus infection at an Italian dental clinic: portrait of the pilot study population. New Microbiol. 2019:42:133-8.

\section{Publisher's Note}

Springer Nature remains neutral with regard to jurisdictional claims in published maps and institutional affiliations.
Ready to submit your research? Choose BMC and benefit from:

- fast, convenient online submission

- thorough peer review by experienced researchers in your field

- rapid publication on acceptance

- support for research data, including large and complex data types

- gold Open Access which fosters wider collaboration and increased citations

- maximum visibility for your research: over $100 \mathrm{M}$ website views per year

At BMC, research is always in progress.

Learn more biomedcentral.com/submissions 